\title{
Inequality Constrained Kalman Filtering for the Localization and Registration of a Surgical Robot
}

\author{
Stephen Tully, George Kantor, and Howie Choset
}

\begin{abstract}
We present a novel method for enforcing nonlinear inequality constraints in the estimation of a high degree of freedom robotic system within a Kalman filter. Our constrained Kalman filtering technique is based on a new concept, which we call uncertainty projection, that projects the portion of the uncertainty ellipsoid that does not satisfy the constraint onto the constraint surface. A new PDF is then generated with an efficient update procedure that is guaranteed to reduce the uncertainty of the system. The application we have targeted for this work is the localization and automatic registration of a robotic surgical probe relative to preoperative images during image-guided surgery. We demonstrate the feasibility of our constrained filtering approach with data collected from an experiment involving a surgical robot navigating on the epicardial surface of a porcine heart.
\end{abstract}

\section{INTRODUCTION}

Minimally invasive surgery (MIS) has become increasingly popular in medicine due to shortened patient recovery time and reduced risk of infection. The trade-off when operating through a small incision, though, is that a tool cannot be viewed with direct vision, thus a surgeon must use a form of real-time medical imaging [1]-[4] during MIS to navigate a surgical tool to a desired anatomical location.

An alternative method for surgical guidance is to provide a virtualized rendered view of the operation for visual feedback (referred to as image-guided surgery), as in [5]. This method is typically based on registering a tracked surgical device to the coordinate frame of preoperatively reconstructed surface models and then overlaying an image of the device on a rendering of anatomical structures (see our implementation in Fig. 1). In practice, though, the localization and registration of a surgical device can be affected by sensor noise, organs shifting, tissue deformation, and noisy imaging.

In the case of poor registration and localization during image-guided surgery, a surgical tool may appear in a location that is completely unrealistic, for example inside of a cardiac surface model when the intervention is performed outside of the heart. Fig. 1 shows an example of exactly this situation from an experiment that we performed with a highly articulated surgical robot called HARP [6], [7].

To improve image-guidance for more accurate feedback during surgery, we are introducing a new method of constrained Kalman filtering to recursively correct registration and localization parameters for a robotic system with known geometric constraints. This is applicable to image-guided

S. Tully is with the Electrical and Computer Engineering Department at Carnegie Mellon University, Pittsburgh, PA 15213, USA. G. Kantor and $\mathrm{H}$. Choset are with the Robotics Institute at Carnegie Mellon University, Pittsburgh, PA 15213, USA, \{stully@ece, kantoreri, choset@cs\}. cmu . edu.

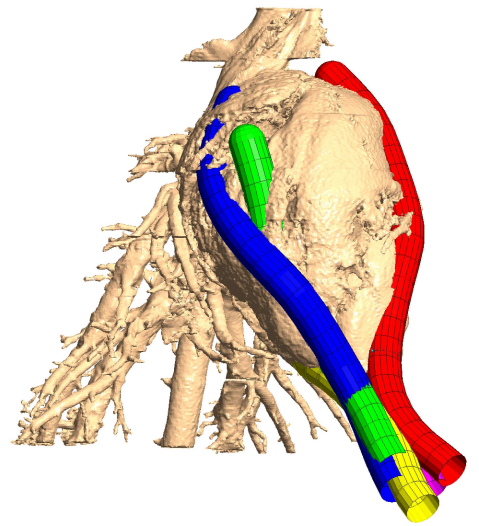

Fig. 1. Our image-guidance system, shown here with multiple paths drawn for the robot. In this image, the robot is shown intersecting the surface model and violating geometric constraints.

surgery because anatomical surface models can indirectly provide information about infeasible robot configurations that we can formulate as constraints when estimating the state of the system in a filtering framework.

The contributions of the work presented in this paper are: 1) the novel use of constrained filtering to correct the localization and registration of a surgical robot, 2) a new constrained filtering algorithm called uncertainty projection, 3) a pseudo-measurement update method to correct the state estimate of a nonlinear system after performing uncertainty projection, and 4) an experimental result that demonstrates the impact this methodology can have in improving the quality of surgical feedback during MIS.

\section{RElAted WORK}

\section{A. Image-Guided Surgery and Registration}

During MIS, a physician requires visual feedback to navigate a surgical tool to a desired anatomical location. Thus, there are many forms of medical imaging that have been adapted for real-time use. For example, fluoroscopy is a form of real-time X-ray that is commonly used [1], MRI and CT [3], [8] have recently been extended to intra-operative use, and 3D ultrasound [9] is becoming a popular alternative. Unfortunately, many of these imaging modalities either have a limited field of view, are incompatible with robotic systems, and/or emit significant radiation.

With image-guided surgery, a tracking device is attached to a surgical tool or robot to continuously determine the tool's pose in 3D space. The information from the tracker is fused with some form of image reconstruction from preoperative or 
live data, such as in [10]. The goal of image-guided surgery is to produce a $3 \mathrm{D}$ visualization that overlays a rendering of the surgical tool alongside the anatomical structures of interest. Commercial examples of this type of sensor-guided feedback are Ensite NavX (St Jude Medical, St Paul, MN, USA) and Carto XP/CartoMerge (Bio-Sense Webster, Diamond Bar, CA, USA), which are used for cardiac mapping and ablation.

To properly fuse pose estimates from a tracker with anatomical surface models, the two frames of reference must be registered. A common method is to use an iterative closest point (ICP) method [11]. Unfortunately, this can be computationally intensive in the case of aligning large point clouds [12]. Another method uses ultrasound to register a surgical tool inside of a heart model using a particle filter [2]. The benefit of our work over these other approaches is that our algorithm is applicable to higher dimensional systems (e.g., a surgical snake robot) and our system will automatically adjust registration and localization parameters during an experiment if something changes.

\section{B. Constrained Filtering}

Constrained filtering is the problem of correcting or constraining the Kalman update or Kalman prediction to account for known constraints on the state vector [13]. In [14], Simon and Chia present a method for equality constrained Kalman filtering in which linear constraints are incorporated into the Kalman filter update by projecting the unconstrained estimate onto the constraint hyperplane. Another popular method is to use a pseudo-measurement approach that simulates a perfect measurement to enforce equality constraints on the Kalman filter update step [15]-[17]. In [18], [19], it was shown that the pseudo-measurement update method is actually equivalent to the state projection method from [14].

Another related problem, which more closely relates to this paper, is inequality constrained Kalman filtering [20][23]. One method for inequality constrained filtering is to simply apply one of the aforementioned equality constrained filtering algorithms in order to shift the estimate back into a feasible state. This process, though, would not place the mean of the filter near the true mean of the feasible region in the state space. Instead, several researchers suggest the use of a PDF truncation method that updates the filter with the mean and covariance of the region of the PDF that lies outside of the constraint hyperplane [20], [21].

\section{UNCERTAINTY PROJECTION}

Inequality constrained filtering seeks to eliminate infeasible states from the PDF of a Kalman filter, which can improve the accuracy of the state estimate and reduce uncertainty. In this section, we will introduce a new algorithm that we call uncertainty projection that can be used to update the PDF of a Gaussian random vector given a set of linear constraints. In Sec. IV, we will then extend our solution to nonlinear constraints.

\section{A. Problem Definition}

Suppose we have a prior PDF of an $M \times 1$ state vector $s$ parameterized by a Gaussian distribution with mean $\hat{s}_{0}$ and covariance $C_{0}$, thus $s \sim \mathcal{N}\left(\hat{s}_{0}, C_{0}\right)$. Also, suppose we have a set of $L$ linear constraints that define feasible states,

$$
\phi_{i}^{T} s \geq \alpha_{i} \quad i=1 \ldots L,
$$

where each $\alpha_{i}$ is a scalar and each $\phi_{i}$ is an $M \times 1$ vector. We note that, if desired, a "less-than" constraint can be defined in the above formulation by negating the $\phi_{i}$ and $\alpha_{i}$ terms.

The objective of our uncertainty projection algorithm is to update the state estimate $\left(\hat{s}_{0}, C_{0}\right)$ given the inequality constraints. Unfortunately, there is no efficient way to apply all of the constraints together. Thus, as in [20], our formulation will apply each constraint in Eq. 1 one-by-one in sequence: thus the estimate $\left(\hat{s}_{i}, C_{i}\right)$ obtained after applying the $i$-th constraint will be used as the new prior when applying the $(i+1)$-th constraint. The result of our algorithm will be a new estimate $\left(\hat{s}_{L}, C_{L}\right)$ that will better approximate the true PDF given the constraints.

\section{B. Algorithm}

To compute $\left(\hat{s}_{i}, C_{i}\right)$ given the associated $i$-th constraint in Eq. 1, the first step is to transform the state space in such a way that the constraint is decoupled from all elements of the transformed state vector except for the first element. This method is similarly used in [20], [21] for PDF truncation. We perform this transformation by defining the random vector,

$$
\begin{aligned}
& y=V W^{-1 / 2} T^{T}\left(s-\hat{s}_{i-1}\right), \\
& y \sim \mathcal{N}\left(0_{M x 1}, I_{M x M}\right),
\end{aligned}
$$

where $T$ and $W$ are obtained from the Jordan canonical decomposition of the prior covariance $\left(C_{i-1}=T W T^{T}\right)$ and $V$ is obtained by using Gram-Schmidt orthogonalization [20], [24] to find the matrix that satisfies,

$$
V W^{1 / 2} T^{T} \phi_{i}=\left[\begin{array}{llll}
\left(\phi_{i}^{T} C_{i-1} \phi_{i}\right)^{1 / 2} & 0 & \ldots & 0
\end{array}\right] .
$$

The significance of this transformation is that $y$ is both zero mean and white and the constraint only applies to the first element of $y$. A new constraint equation can thus be written,

$$
\begin{gathered}
{\left[\begin{array}{cccc}
1 & 0 & \ldots & 0
\end{array}\right] y \geq \beta_{i}} \\
\beta_{i}=\frac{\alpha_{i}-\phi_{i}^{T} \hat{s}_{i-1}}{\left(\phi_{i}^{T} C_{i-1} \phi_{i}\right)^{1 / 2}} .
\end{gathered}
$$

Because the first element of $y$ is the only constrained element of this transformed problem, we have reduced multivariate constrained filtering to a simpler PDF update problem for a scalar Gaussian random variable (mean equal to zero and variance equal to one) that is subject to a scalar constraint.

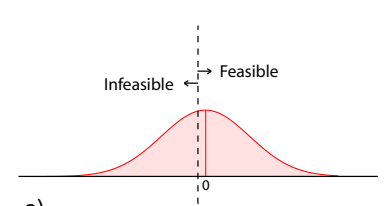

a)

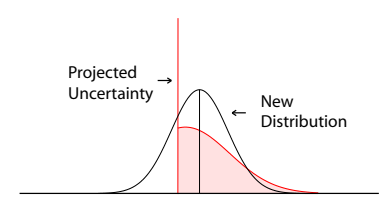

b)
Fig. 2. In (a), a normally distributed random variable is shown with a scalar constraint that defines the feasible and infeasible regions. In (b), we show our uncertainty projection method which projects the tail of the Gaussian onto the constraint and then computes the new mean and variance. 

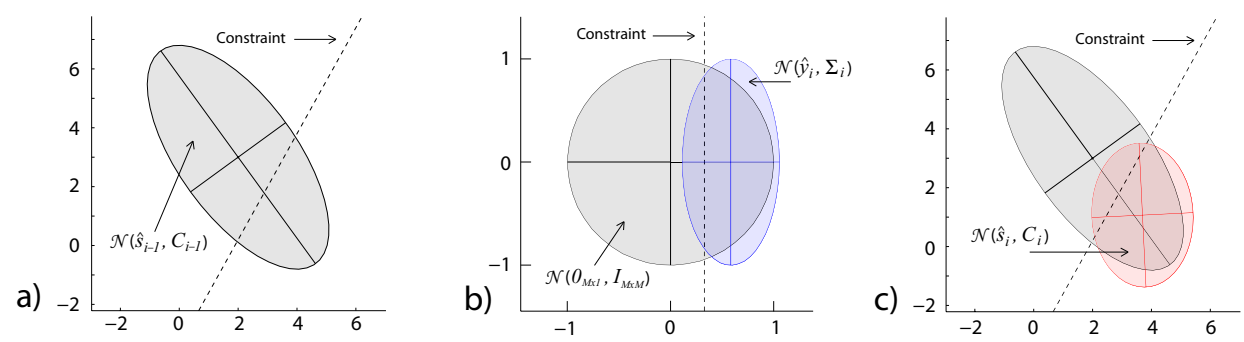

Fig. 3. Shown here is an example of the uncertainty projection method we use for updating the mean and variance. In (a) we show the prior distribution and the constraint. In (b) we show the decoupled transformed state and its uncertainty projected solution. In (c) we show the result from (b) transformed back into the original state space.

To update this one-dimensional PDF, we project the portion of the probability mass that is less than the constraint onto the constraint itself and then compute the new mean and variance, see Fig. 2. The motivation for computing the new estimate this way is that it best preserves the contribution of the probability mass less than the constraint. To perform this uncertainty projection update, we can write the definition of expectation, as follows,

$$
\begin{aligned}
\mu_{i} & =\int_{-\infty}^{\beta_{i}} \frac{\beta_{i}}{\sqrt{2 \pi}} e^{\frac{-z^{2}}{2}} d z+\int_{\beta_{i}}^{\infty} \frac{z}{\sqrt{2 \pi}} e^{\frac{-z^{2}}{2}} d z \\
\sigma_{i}^{2} & =\int_{-\infty}^{\beta_{i}} \frac{\left(\beta_{i}-\mu\right)^{2}}{\sqrt{2 \pi}} e^{\frac{-z^{2}}{2}} d z+\int_{\beta_{i}}^{\infty} \frac{(z-\mu)^{2}}{\sqrt{2 \pi}} e^{\frac{-z^{2}}{2}} d z .
\end{aligned}
$$

This formulation involves two integrals: one that is associated with the infeasible region and one that is associated with the feasible region, with $\beta_{i}$ being the point of the constraint. The solution that we have derived by evaluating these integrals,

$$
\begin{aligned}
\gamma_{i} & =-2 \pi-2 \beta_{i} e^{\frac{-\beta_{i}^{2}}{2}} \sqrt{2 \pi} \\
\mu_{i} & =\frac{1}{\sqrt{2 \pi}} e^{\frac{-\beta_{i}^{2}}{2}}+\frac{1}{2} \beta_{i}\left[1+\operatorname{Erf}\left(\frac{\beta_{i}}{\sqrt{2}}\right)\right] \\
\sigma_{i}^{2} & =\frac{1}{4 \pi}\left[-2 e^{-\beta_{i}^{2}}+\left(2+\beta_{i}^{2}\right) \pi+\gamma_{i} \operatorname{Erf}\left(\frac{\beta_{i}}{\sqrt{2}}\right)-\beta_{i}^{2} \pi \operatorname{Erf}\left(\frac{\beta_{i}}{\sqrt{2}}\right)^{2}\right],
\end{aligned}
$$

can then be transformed back into the original state space by reversing the transformation that originally whitened the noise to decouple the elements of the state vector,

$$
\begin{aligned}
& \hat{y}_{i}=\left[\begin{array}{llll}
\mu_{i} & 0 & \ldots & 0
\end{array}\right]^{T} \\
& \Sigma_{i}=\operatorname{diag}\left(\sigma_{i}^{2}, 1, \ldots, 1\right) \\
& \hat{s}_{i}=T W^{1 / 2} V^{T} \hat{y}_{i}+\hat{s}_{i-1} \\
& C_{i}=T W^{1 / 2} V^{T} \Sigma_{i} V W^{1 / 2} T^{T} .
\end{aligned}
$$

In summary, through the update process above, the mean $\hat{s}_{i}$ and covariance $C_{i}$ are updated recursively from $\left(\hat{s}_{i-1}, C_{i-1}\right)$ given the $i$-th constraint equation in Eq. 1.

The example in Fig. 3 illustrates our uncertainty projection method. In Fig. 3-(a), a constraint tells us that the true state must lie to the right of the dotted line. The algorithm transforms the state according to Eq. 2, the result of which is shown in Fig. 3-(b), and a new estimate, labeled as $\left(\hat{y}_{i}, \Sigma_{i}\right)$, is computed using Eq. 4. Lastly, the estimate is transformed back to the original state space, resulting in an updated estimate, shown in Fig. 3-(c).
The significance of the example in Fig. 3 is that the resulting uncertainty ellipse qualitatively bounds the region of the PDF that is deemed feasible by the constraint equation while successfully discarding the majority of the infeasible region of the PDF. We note that our efficient algorithm can be used for any set of linear constraints imposed on a multidimensional Gaussian distribution.

\section{NONLINEAR INEQUALITy CONSTRAined FILTERING}

In this section, we will introduce a second algorithm that will extend our uncertainty projection algorithm to systems with nonlinear constraints, which is particularly applicable to our surgical application.

\section{A. Problem Definition}

Suppose that a Kalman filter is estimating a system with an $N \times 1$ state vector $x_{k}$. After each prediction, the Kalman filter produces an estimate $\left(\hat{x}_{k \mid k-1}, P_{k \mid k-1}\right)$ and after each correction, the Kalman filter produces an estimate $\left(\hat{x}_{k \mid k}, P_{k \mid k}\right)$. The constrained filtering problem that we define here is to update the corrected estimate $\left(\hat{x}_{k \mid k}, P_{k \mid k}\right)$ given a set of $L$ constraints. This is similar to our formulation in Sec. III, except suppose now that the constraints are nonlinear,

$$
\phi_{i}^{T} a\left(x_{k}\right) \geq \alpha_{i} \quad i=1 \ldots L .
$$

In this formulation, we assume that a known function $a\left(x_{k}\right)$ exists that will map the state vector to a space in which the constraints are linear, which we call the constraint-space. For many applications, the constraint-space should be chosen to be the workspace of the robot, for this is where the system will be limited by geometric constraints.

\section{B. Algorithm}

The first step in our constrained filtering algorithm is to project the Kalman filter estimate $\left(\hat{x}_{k \mid k}, P_{k \mid k}\right)$ into the constraint-space with the following projection,

$$
\hat{s}_{0}=a\left(\hat{x}_{k \mid k}\right), \quad C_{0}=A_{k} P_{k \mid k} A_{k}^{T},
$$

where $A_{k}$ is the Jacobian of the nonlinear function $a\left(x_{k}\right)$ linearized about the current estimate $\hat{x}_{k \mid k}$.

After performing this projection, the problem has been cast into a form that is solvable with our uncertainty projection algorithm for linear constraints, which we discussed in Sec. III. After plugging the estimate $\left(\hat{s}_{0}, C_{0}\right)$ into our uncertainty 


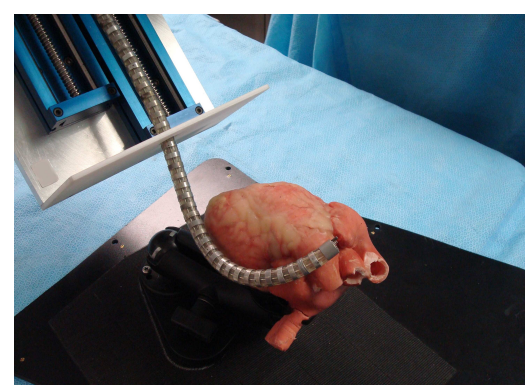

Fig. 4. This is the HARP robot that we used for our constrained filtering experiments.

projection algorithm along with the constraints, the result would be the updated estimate $\left(\hat{s}_{L}, C_{L}\right)$. This result still lies in the constraint-space, but now has reduced uncertainty and is likely a better estimate of the PDF given the constraints.

To update the actual Kalman estimate, we need to modify the PDF so that its projection into the constraint-space corresponds to the new estimated uncertainty in the constraintspace. In most applications, unfortunately, the dimension of the constraint-space will be lower than that of the state space. This means that such a projection is not uniquely defined nor easy to compute. To solve this problem, we introduce a covariance update and a mean update based on applying a pseudo-measurement. Our algorithm is described in Alg. 1.

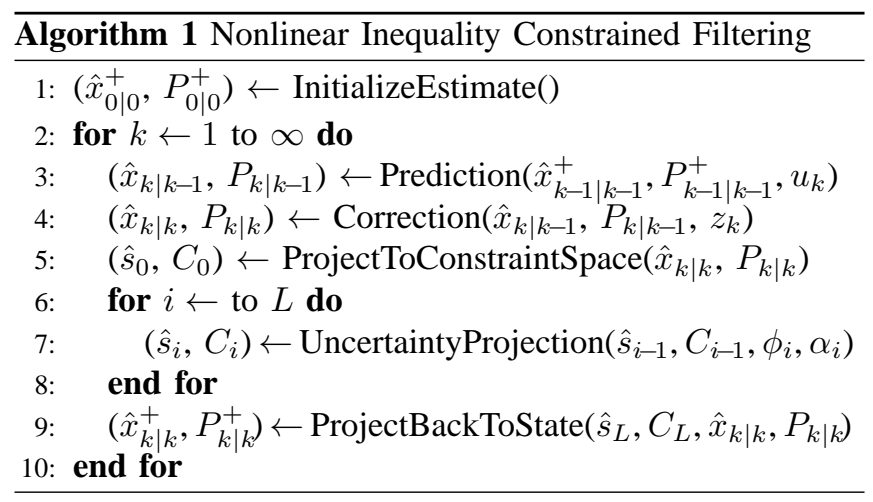

\section{Covariance Update}

To compute the updated covariance matrix of the state, we would like to find the matrix $P_{k \mid k}^{+}$that, when projected into the constraint-space, is equal to the uncertainty projected covariance $C_{L}$, thus,

$$
A_{k} P_{k \mid k}^{+} A_{k}^{T}=C_{L}
$$

Unfortunately, there are possibly infinite solutions to this problem when the dimension of the state space is higher than the dimension of the constraint-space.

Our solution is to pretend that there is a pseudomeasurement in the constraint-space applied to the state that we can define in such a way to update the PDF so that its projection into the constraint space is the uncertainty projected solution. The motivation for using a pseudo-measurement for this update process is that it lowers the dimension of the problem that we need to solve to update the covariance.

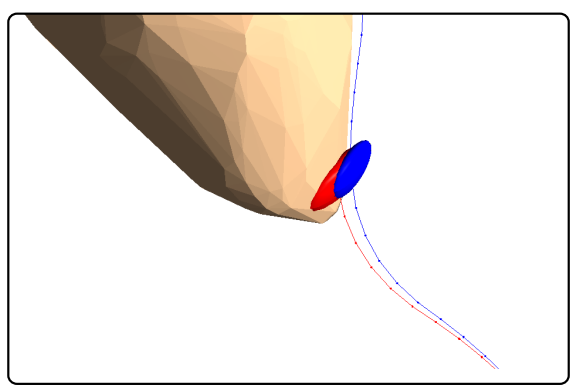

Fig. 5. This is an example of our constrained filtering method correcting the estimated configuration of the HARP surgical robot based on the constraints imposed by the surface model.

We first force the resulting covariance of the pseudomeasurement update to satisfy Eq. 6. To do this, we take the standard Kalman covariance update and then both left and right multiply by $A_{k}$,

$$
\begin{aligned}
& A_{k} P_{k \mid k}^{+} A_{k}^{T}= \\
& \quad A_{k} P_{k \mid k} A_{k}^{T}-A_{k} P_{k \mid k} A_{k}^{T}\left(A_{k} P_{k \mid k} A_{k}^{T}+R\right)^{-1} A_{k} P_{k \mid k} A_{k}^{T},
\end{aligned}
$$

which can be simplified, as follows,

$$
C_{L}=C_{0}-C_{0}\left(C_{0}+R\right)^{-1} C_{0}
$$

The unknown in Eq. 7 is the covariance of the pseudomeasurement $R$. The closed form solution that will determine the matrix $R$ that satisfies Eq. 6 and Eq. 7 is,

$$
R=\left(C_{0}^{-1}-C_{0}^{-1} C_{L} C_{0}^{-1}\right)^{-1}-C_{0} .
$$

If we plug this measurement covariance into the standard Kalman covariance update equation, we will have an efficient equation for updating the covariance in the state space from the estimated covariance $C_{L}$ in the constraint-space that was obtained from uncertainty projection,

$$
\begin{aligned}
P_{k \mid k}^{+} & =P_{k \mid k}-P_{k \mid k} A_{k}^{T}\left(A_{k} P_{k \mid k} A_{k}^{T}+R\right)^{-1} A_{k} P_{k \mid k} \\
& =P_{k \mid k}-P_{k \mid k} A_{k}^{T}\left(C_{0}^{-1}-C_{0}^{-1} C_{L} C_{0}^{-1}\right) A_{k} P_{k \mid k} .
\end{aligned}
$$

It is important to note that Eq. 9 represents the prior covariance $P_{k \mid k}$, which is a positive definite matrix, subtracted by another positive definite matrix. For this reason, the constrained filtering approach that we are introducing here is guaranteed to reduce the uncertainty in the system as long as $C_{L}$ is unequal to $C_{0}$. This will always be the case with our uncertainty projection method from Sec. III.

\section{Mean Update}

To compute the updated mean vector $\hat{x}_{k \mid k}^{+}$, we would like to find the appropriate state that, when projected into the constraint-space, is equal to the newly computed uncertainty projected estimate $\hat{s}_{L}$,

$$
a\left(\hat{x}_{k \mid k}^{+}\right)=\hat{s}_{L}
$$

We will again use a pseudo-measurement method to update the mean of the Kalman filter. Assuming the same pseudomeasurement covariance $R$ from Eq. 8, we can write a 

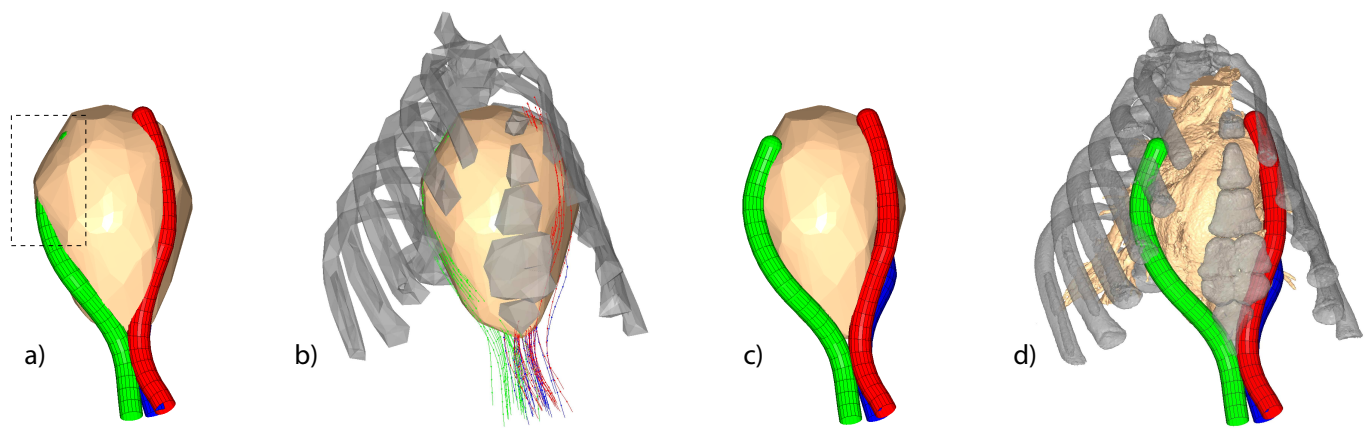

Fig. 6. Experiment I: (a) shows an initial estimate of three paths performed during an experiment on a porcine subject that violates constraints, (b) depicts the many hypotheses we are testing, and (c),(d) show the result of our filtering algorithm that has corrected the estimate.

Kalman gain matrix, as follows,

$$
\begin{aligned}
K & =P_{k \mid k} A_{k}^{T}\left(A_{k} P_{k \mid k} A_{k}^{T}+R\right)^{-1} \\
& =P_{k \mid k} A_{k}^{T}\left(C_{0}^{-1}-C_{0}^{-1} C_{L} C_{0}^{-1}\right)
\end{aligned}
$$

We then force the resulting mean of the pseudo-measurement update to satisfy Eq. 10. To do this, we take the standard Kalman mean update equation,

$$
\hat{x}_{k \mid k}^{+}=\hat{x}_{k \mid k}+K\left(z-\hat{s}_{0}\right)
$$

and then cast both sides into the constraint-space (this assumes linearization),

$$
\hat{s}_{L}=\hat{s}_{0}+A_{k} K\left(z-\hat{s}_{0}\right) .
$$

The unknown in Eq. 11 is the pseudo-measurement vector $z$. The closed form solution that will compute the vector $z$ that satisfies Eq. 10 and Eq. 11 is,

$$
z=\hat{s}_{0}+\left(A_{k} K\right)^{-1}\left(\hat{s}_{L}-\hat{s}_{0}\right) .
$$

If we plug this measurement vector into the standard Kalman mean update equation, we will have an efficient equation for updating the mean in the state space from the mean $\hat{s}_{L}$ in the constraint-space obtained from uncertainty projection,

$$
\hat{x}_{k \mid k}^{+}=\hat{x}_{k \mid k}+K\left(A_{k} K\right)^{-1}\left(\hat{s}_{L}-\hat{s}_{0}\right) .
$$

In summary, the mean update presented here uses a pseudomeasurement correction to modify the Kalman estimate with the result from our uncertainty projection algorithm.

\section{SURGICAL REgISTRATION AND LOCALIZATION}

The robot we are using for experiments is a highly articulated robotic probe (HARP). The HARP was originally presented in [6], [7], [25]. The advantage of the HARP is that it has the stability of a rigid device and the maneuverability of a flexible tool. The device is shown in Fig. 4.

\section{A. Experiment I}

We have performed several image-guidance experiments on porcine subjects where we obtained preoperative CT images and then processed preoperative surface models. In one experiment, we used a magnetic tracker (NDI Aurora from Northern Digital Inc, Waterloo, Ontario, Canada) to track the distal tip of the robot to collect data for the shape/configuration of the robot when driving along three paths around the epicardial surface of the porcine heart.

In Fig. 6-(a), we show the initial estimate of the three paths based on an initial fiducial-based registration procedure. Within the rectangle in Fig. 6-(a), we show that the initial estimate is erroneous and clearly intersects the heart surface.

To perform constrained filtering for this problem, we first sample over a set of parameters that define the registration of the robot to the surface model (see Fig. 6-(b)). For each hypothesis, we perform the inequality constrained filtering algorithm that we have presented in this paper. The constraint equations we formulate at each time step are based on computing the positions of various points along the robot paths using a forward kinematic model and then intersecting the point's projected uncertainty ellipse in the workspace with the heart surface model. In Fig. 5, we show an example of a constraint update changing the estimated path to agree with the constraint. The parameters we estimate for each hypothesis are the joint angles of the links of the robot along with the pose of the initial link, for each of the three paths.

The result, after applying our novel constrained filtering approach and choosing the most likely hypothesis (in this case, the one that deviates the least from the initial estimate), is shown in Fig. 6-(c). The final result shown with detailed surface models is shown in Fig. 6-(d). We note that while this experimental result was post-processed after the fact, the algorithm we are introducing is defined in such a way that it could be efficiently applied during a live experiment.

\section{B. Experiment II}

In a second experiment, we tested our constrained filtering algorithm with data from a bench-top experiment. For this experiment, we used a trakSTAR ${ }^{\mathrm{TM}}$ EM sensor (Ascension Technologies, Burlington, VT, USA). We configured the robot in 5 different paths around a rubber heart model and collected shape data for each of the paths. We obtained ground truth alignment by carefully registering the tracker to the surface model with fiducial markers. To simulate the errors that would occur in a surgical trial, we added extra noise to the ground truth data to achieve an erroneous initial estimate (see the initial estimate in Fig. 7-(a)).

As before, we sampled over the registration parameters and for each hypothesis, we recursively ran a Kalman filter 

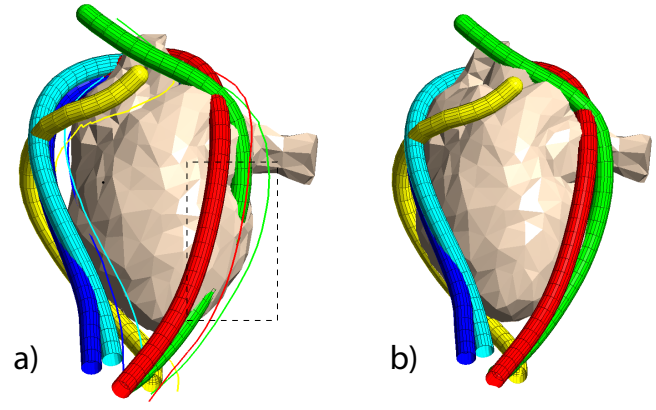

Fig. 7. Experiment II: in (a) we show the initialized estimate for the registration and configuration of the HARP robot for 5 different paths around the heart model. In (b), we show the corrected estimate that we achieved with our constrained filtering method.

that estimated the joint angles and the pose of the initial link. We used our constrained filtering algorithm to update an estimate when a point along a robot path intersected the surface model, thus violating a constraint. The result of our constrained filtering algorithm is shown in Fig. 7-(b). The initial average error (before constraining the estimate) of the end effector positions for the 5 paths was $16.13 \mathrm{~mm}$. After applying our algorithm, the average error of the end effector positions was $2.19 \mathrm{~mm}$ versus ground truth. This demonstrates the gain in accuracy we can achieve by leveraging known geometric constraints in the environment.

\section{CONCLUSION}

The work presented here is a novel method for enforcing nonlinear inequality constraints in the estimation of a high degree of freedom robotic system within a Kalman filter. We have introduced a new algorithm based on uncertainty projection and have developed a constraint update process based on a pseudo-measurement correction step.

The application we discussed in this paper is the localization and automatic registration of a robotic surgical probe. We have shown promising results from data collected with bench-top and animal experiments. We have shown that our method is particularly beneficial for correcting registration error in the case of known geometric constraints imposed by preoperative surface models.

It is worth noting that our work has assumed that the tissue models that we are registering to are inherently rigid structures. For surgical applications, this is an assumption we must make due to the lack of more representative preoperative models. Future work on this subject will relax this assumption.

\section{REFERENCES}

[1] L. Joskowicz, M. Sati, and L. Nolte, "Fluoroscopy-based navigation in computer-aided orthopaedic surgery," in Proceedings of the IFAC Conf. Mechatronic Systems, 2000.

[2] A. B. Koolwal, F. Barbagli, C. Carlson, and D. Liang, "An ultrasoundbased localization algorithm for catheter ablation guidance in the left atrium," The International Journal of Robotics Research, vol. 29, no. 6, pp. 643-665, May 2010.

[3] R. Omary, J. Green, B. Schirf, Y. Li, J. Finn, and D. Li, "Realtime magnetic resonance imaging-guided coronary catheterization in swine," Circulation, vol. 107, no. 21, pp. 2656-2659, 2003.
[4] M. Mack, "Minimally Invasive and Robotic Surgery," The Journal of the American Medical Association, vol. 285, no. 5, pp. 568-572, 2001.

[5] W. Wein, A. Khamene, D. Clevert, O. Kutter, and N. Navab, "Simulation and fully automatic multimodal registration of medical ultrasound," in Medical Image Computing and Computer-Assisted Intervention (MICCAI), 2007, pp. 136-143.

[6] A. Degani, H. Choset, A. Wolf, and M. Zenati, "Highly articulated robotic probe for minimally invasive surgery," in Robotics and $\mathrm{Au}$ tomation, 2006. ICRA 2006. Proceedings 2006 IEEE International Conference on, May 2006, pp. 4167-4172.

[7] A. Degani, H. Choset, A. Wolf, T. Ota, and M. Zenati, "Percutaneous intrapericardial interventions using a highly articulated robotic probe," in IEEE/RAS-EMBS International Conference on Biomedical Robotics and Biomechatronics, Feb 2006, pp. 7-12.

[8] R. Manzke, V. Reddy, S. Dalal, A. Hanekamp, V. Rasche, and R. Chan, "Intra-operative volume imaging of the left atrium and pulmonary veins with rotational x-ray angiography," in Medical Image Computing and Computer-Assisted Intervention (MICCAI), 2006, pp. 604-611.

[9] W. Zhang, R. Rohling, and D. K. Pai, "Surface extraction with a threedimensional freehand ultrasound system," Ultrasound in Medicine and Biology, vol. 30, no. 11, pp. 1461-1473, 2004.

[10] K. Cleary, H. Zhang, N. Glossop, E. Levy, B. Wood, and F. Banovac, "Electromagnetic tracking for image-guided abdominal procedures: Overall system and technical issues," in Engineering in Medicine and Biology Society, 2005. IEEE-EMBS 2005. 27th Annual International Conference of the, 2005, pp. 6748-6753.

[11] H. Zhong, T. Kanade, and D. Schwartzman, "Sensor guided ablation procedure of left atrial endocardium," in Medical Image Computing and Computer-Assisted Intervention (MICCAI), 2005, pp. 1-8.

[12] J. Dong, H. Calkins, S. Solomon, S. Lai, D. Dalal, A. Lardo, E. Brem, A. Preiss, R. Berger, H. Halperin, and T. Dickfeld, "Integrated electroanatomic mapping with three-dimensional computed tomographic images for real-time guided ablations," Circulation, vol. 113, pp. 186194, 2006.

[13] D. Simon, "Kalman filtering with state constraints: a survey of linear and nonlinear algorithms," Control Theory Applications, IET, vol. 4, no. 8, pp. 1303-1318, August 2010.

[14] D. Simon and T. Chia, "Kalman filtering with state equality constraints," IEEE Transactions Aerosp. Electron. Systems, vol. 39, no. 1, pp. 128-136, January 2002.

[15] M. Tahk and J. Speyer, "Target tracking problems subject to kinematic constraints," IEEE Transactions Autom. Control, vol. 35, no. 3, pp. 324-326, March 1990.

[16] A. Alouani and W. Blair, "Use of a kinematic constraint in tracking constant speed, maneuvering targets," IEEE Trans. Autom. Control, vol. 38, no. 7, pp. 1107-1111, July 1993.

[17] L. Wang, Y. Chiang, and F. Chang, "Filtering method for nonlinear systems with constraints," Control Theory and Applications, IEEE Proceedings, vol. 149, no. 6, pp. 525-531, November 2002.

[18] B. Teixeira, J. Chandrasekar, L. Torres, L. Aguirre, and D. Bernstein, "State estimation for equality-constrained linear systems," in Decision and Control, IEEE Conference on, December 2007, pp. 6220-6225.

[19] N. Gupta, "Kalman filtering in the presence of state space equality constraints," in Chinese Control Conf., December 2007, pp. 107-113.

[20] D. Simon and D. L. Simon, "Constrained kalman filtering via density function truncation for turbofan engine health estimation," Int. Journal of Systems Science, vol. 41, no. 2, pp. 159-171, February 2010.

[21] N. Shimada, Y. Shirai, Y. Kuno, and J. Miura, "Hand gesture estimation and model refinement using monocular camera - ambiguity limitation by inequality constraints," in Proc. The 3rd Int. Conference on Automatic Face and Gesture Recognition, 1998, pp. 268-273.

[22] B. O. Teixeira, L. A. Trres, L. A. Aguirre, and D. S. Bernstein, "On unscented kalman filtering with state interval constraints," Journal of Process Control, vol. 20, no. 1, pp. 45-57, 2010.

[23] P. Vachhani, S. Narasimhan, and R. Rengaswamy, "Robust and reliable estimation via unscented recursive nonlinear dynamic data reconciliation," Journal of Proc. Control, vol. 16, no. 10, pp. 1075-1086, 2006.

[24] T. Moon and W. Stirling, Math. Methods and Algorithms for Signal Processing. New Jersey: Prentice Hall, Upper Saddle River, 2000.

[25] T. Ota, A. Degani, D. Schwartzman, B. Zubiate, J. McGarvey, H. Choset, and M. A. Zenati, "A novel highly articulated robotic surgical system for epicardial ablation," in Engineering in Medicine and Biology Society, 2008. EMBS 2008. 30th Annual International Conference of the IEEE, Aug 2008, pp. 250-253. 